\title{
Compressibility of tableting materials and properties of tablets with glyceryl behenate
}

\author{
JITKA MUŽÍKOVÁ ${ }^{*}$ \\ SANDRA MUCHOVÁ ${ }^{1}$ \\ ALENA KOMERSOVÁ ${ }^{2}$ \\ VÁCLAV LOCHA Ř ${ }^{2}$ \\ ${ }^{1}$ Department of Pharmaceutical \\ Technology, Charles University in \\ Prague, Faculty of Pharmacy in \\ Hradec Králové, Czech Republic \\ ${ }^{2}$ Department of Physical Chemistry \\ University of Pardubice, Faculty of \\ Chemical Technology, Pardubice \\ Czech Republic
}

Accepted October 14, 2014

\begin{abstract}
The paper studies the compressibility of directly compressible tableting materials with dry binders, spray-dried lactose and microcrystalline cellulose, and glyceryl dibehenate at various concentrations. Compressibility was evaluated by means of the energy profile of compression and tensile strength of tablets. Release rate of the active ingredient, salicylic acid, from the tablets was also examined. In the case of microcrystalline cellulose, a higher concentration of glyceryl dibehenate increased the strength of tablets, while this did not occur in the case of spray-dried lactose. Increasing concentration of glyceryl dibehenate prolonged the release of salicylic acid; however, no statistically significant difference was found compared to the type of the dry binder used.
\end{abstract}

Keywords: lipophilic matrix tablet, glyceryl dibehenate, salicylic acid, energy profile of compression, tensile strength of tablets, dissolution profile

Lipophilic matrix systems have recently become popular due to their cheap and easy preparation and chemical inertness. They contain various fat alcohols, waxes and fats, e.g., cetyl alcohol, glyceryl dibehenate, and carnauba wax $(1,2)$. These systems are primarily used for prolonged release of very easily water-soluble drugs. When a matrix tablet is placed in a dissolution medium, flaws, canals and pores are produced on its surface, through which the drug is released from the surface and subsequently also from deeper parts of the matrix (3). A frequently employed substance for the preparation of lipophilic matrix tablets is glyceryl dibehenate in the form of the brand product Compritol ${ }^{\circledR} 888$ ATO. This substance, at concentrations of $1-3 \%$, is also used in tablets as a lubricant, while for prolonged release a concentration above $10 \%$ is necessary (4). The advantages of matrix tablets with glyceryl dibehenate include their possible manufacture by direct compression as well as tableting after granulation, high resistance against physiological conditions, $\mathrm{pH}$ independence of drug release; moreover, the active ingredient is released by diffusion and the burst-out effect is minimized (5). The aim of this paper was to study the compressibility of tableting materials with different types of dry binders and various concentrations

\footnotetext{
*Correspondence; e-mail: muzikova@faf.cuni.cz
} 
of glyceryl dibehenate by means of the energy profile of the compression process and tensile strength of tablets. Release rate of the active ingredient, salicylic acid, from lipophilic matrix tablets was also evaluated.

\section{EXPERIMENTAL}

\section{Materials}

Spray-dried lactose Flowlac ${ }^{\circledR} 100$ was supplied by Meggle Pharma (Germany), microcrystalline cellulose Microcel ${ }^{\circledR}$ MC-102 by Blanver (Brazil). Glyceryl dibehenate Compritol ${ }^{\circledR}$ 888 ATO was obtained from Gattefossé (France) and sodium stearyl fumarate (Lubripharm ${ }^{\circledR}$ SSF) from SPI Pharma (France). Salicylic acid was from JQC (Huayin) Pharmaceutical Co., Ltd. (China).

\section{Preparation of tableting materials}

Altogether, six tableting materials of different composition were prepared and tested (Table I). Furthermore, tableting materials of the same composition but without salicylic acid were prepared, from which subsequently tablets were compressed and served as blind samples for the dissolution test.

Substances were mixed in a mixing cube KB $15 S$ (Erweka GmbH, Germany). The dry binder was first mixed with the active ingredient for 5 minutes and then glyceryl dibehenate was added for further 5 minutes. The last ingredient added was a lubricant, with which the mixture was mixed for 2.5 minutes. The mixing speed was $17 \mathrm{rpm}$. The total mass of prepared tableting materials was $30 \mathrm{~g}$. Mixtures without salicylic acid were mixed in the same manner, omitting the step of salicylic acid addition; their mass was $10 \mathrm{~g}$.

\section{Preparation of tablets and energy evaluation of the compression process}

Tablets were compressed using the material testing equipment T1-FRO 50 TH.A1K Zwick/Roell (Zwick GmbH\&Co, Germany) by means of a special die with a lower and an

Table I. Composition of tableting materials (\%)

\begin{tabular}{lccccc}
\hline $\begin{array}{l}\text { Tableting } \\
\text { material }\end{array}$ & $\begin{array}{c}\text { Flowlac }^{\circledR} \\
100\end{array}$ & $\begin{array}{c}\text { Microcel }^{\circledR} \\
\text { MC-102 }\end{array}$ & $\begin{array}{c}\text { Salicylic } \\
\text { acid }\end{array}$ & $\begin{array}{c}\text { Compritol }^{\circledR} \\
888 \text { ATO }\end{array}$ & $\begin{array}{c}\text { Lubripharm }^{\circledR} \\
\text { SSF }\end{array}$ \\
\hline MCC $+10 \%$ C & - & 64 & 25 & 10 & 1 \\
MCC $+15 \%$ C & - & 59 & 25 & 15 & 1 \\
MCC + 20 \% C & - & 54 & 25 & 20 & 1 \\
FL + $10 \%$ C & 64 & - & 25 & 10 & 1 \\
FL $+15 \%$ C & 59 & - & 25 & 15 & 1 \\
FL $+20 \%$ C & 54 & - & 25 & 20 & 1 \\
\hline
\end{tabular}

MCC - Microcel ${ }^{\circledR}$ MC-102

C - Compritol ${ }^{\circledR} 888$ ATO

FL - Flowlac ${ }^{\circledR} 100$ 
upper punch. The rate of compaction was $40 \mathrm{~mm} \mathrm{~min}^{-1}$, pre-load was $2 \mathrm{~N}$, and the rate of pre-load $2 \mathrm{~mm} \mathrm{~s}^{-1}$. The tablets were of cylindrical shape without facets, with 13-mm diameter and $0.5000 \pm 0.0010 \mathrm{~g}$ mass. Tableting materials with two dry binders were compressed by different compression forces, because the spray-dried lactose was the least compressible. The employed compression forces for tableting materials with microcrystalline cellulose were 4, 5, and 6, for the mixtures with spray-dried lactose 12,14 , and $16 \mathrm{kN}$. Ten tablets were compressed at each compression force. The computer program testXpert V 9.01 (Zwick GmbH\&Co, Germany) simultaneously recorded the energy process of compression by means of the »force-displacement « record and numerically evaluated the energy balance of compression, i.e., the energy of precompression $E_{1}$, energy of plastic deformation $E_{2}$, energy of elastic deformation $E_{3}$, total energy $E_{\max }$, which is the sum of all energies, and plasticity $(6,7)$. Tablets for dissolution made from all tableting materials were also compressed; the employed compression force for the mixtures of microcrystalline cellulose with 10 and $20 \%$ glyceryl dibehenate was $5 \mathrm{kN}$, for the mixture of microcrystalline cellulose and $15 \%$ glyceryl dibehenate $6 \mathrm{kN}$, for the mixtures of spray-dried lactose with 10 and $20 \%$ glyceryl dibehenate $12 \mathrm{kN}$, for the mixture of spray-dried lactose with 15 $\%$ glyceryl dibehenate $15 \mathrm{kN}$. These different compression forces were used so that all tablets may possess approximately the same tensile strength in the range of $0.78-0.88 \mathrm{MPa}$. The number of tablets compressed for dissolution was six.

\section{Measurement of the tensile strength of tablets}

Tensile strength of tablets was measured in 10 tablets no sooner than 24 hours after compression (tablets were stored in plastic tubes). Measurements were performed using a Schleuniger apparatus (Dr. Schleuniger Pharmatron AG, Switzerland), which measures the diameter and height of tablets with a precision of $0.01 \mathrm{~mm}$ and destruction force in $\mathrm{N}$. Tensile strength of tablets was subsequently calculated according to the equation (Eq. 1) (8):

$$
P=2 F /(\pi d h)
$$

where $P$ is tensile strength of the tablet in $\mathrm{MPa}, F$ is destruction force in $\mathrm{N}, d$ is tablet diameter in $\mathrm{mm}, h$ is the tablet height in $\mathrm{mm}$.

\section{Dissolution testing}

Release of the active ingredient, salicylic acid, was always tested from 6 tablets from each tableting material. Tablets were prepared so that all of them had tensile strength within the range from $0.78-0.88 \mathrm{MPa}$. Dissolution testing was performed using the rotating basket method according to the European Pharmacopoeia 7 (9) on a dissolution unit Sotax AT 7 smart (Sotax AG Basel, Switzerland). The test was carried out under sink conditions. Dissolution medium was $900 \mathrm{~mL}$ of purified water tempetured to $37^{\circ} \mathrm{C}$. The rotation rate of the basket was $100 \mathrm{rpm}$, and at one-hour intervals samples of $3 \mathrm{~mL}$ were withdrawn, and then this volume was replaced with pure medium. The content of salicylic acid was determined spectrophotometrically at $270 \mathrm{~nm}$ (spectrophotometer Specord 205, Analytic Jena, Germany) and the program WinAspect (Analytic Jena, Germany). Absorbance was measured vs. a blind sample, which was obtained by dissolution of tablets of the corresponding 
composition but without salicylic acid. Measurements were repeated five times and subsequently averaged. The amount of released active ingredient was determined by means of the calibration curve for salicylic acid.

\section{Mathematical and statistical processing of results}

The results for tensile strengths and dissolution of tablets were statistically processed by means of the computer programmes Excel and Qcexpert (TriloByte Statistical Software, s.r.o., Czech Republic). The values of energies and plasticity were statistically processed by the computer programme testXpert V 9.01 (Zwick GmbH\&Co, Germany) during compaction. In the case of similar significance of values, an unpaired $t$-test at a level of significance of 0.05 was employed. Experimental dissolution data were mathematically processed and statistically evaluated by means of the computer programs GraphPad Prism (GraphPad Software, Inc., USA) and Origin 9 Pro (OriginLab Corporation, USA). Three mathematical models were used for the analysis of dissolution profiles. Coefficient of determination $R^{2}$ and residual sum of squares $R S S$ were used for comparison of dissolution profiles.

\section{RESULTS AND DISCUSSION}

\section{Compressibility of tableting materials}

Compressibility of tableting materials was evaluated by means of the energy profile of the compression process and on the basis of the test of tensile strength of tablets. The individual components of energy balance of compression are listed in Table II. Adjustment of compression forces was thus controlled by the adjustment of tensile strength of tablets within the optimal range of tablet strength, $0.56-1.12 \mathrm{MPa}(10)$. Poorer compressibility of spray-dried lactose is evident from the values of plasticity $(45.0-58.3 \%)$, which are markedly lower than the values for microcrystalline cellulose (81.7-87.9\%). Excellent plastic deformability, which is also the compression mechanism of microcrystalline cellulose, is generally known, in contrast to spray-dried lactose where the mechanism of compression by fragmentation of particles prevails (11). Values in Table II show that the values of all energies in tableting materials with both types of dry binders increase with increasing compression force, plasticity decreases due to a decreasing number of pores in the compact, more markedly decreasing in the case of tableting materials with spray-dried lactose (Flowlac 100). Addition of glyceryl dibehenate to the tableting material with microcrystalline cellulose decreases the total energy of compression primarily due to a decrease in the precompression energy, because here it acts also as a lubricant, and due to the energy of plastic deformation; the energy of elastic deformation is not influenced. The total energy of compression of tableting materials with spray-dried lactose is not markedly influenced by addition of glyceryl dibehenate. Plasticity of tableting materials with both dry binders decreases due to addition of glyceryl dibehenate, more markedly than in the case of spraydried lactose.

Addition of glyceryl dibehenate exerts different impacts on the strength of tablets in relation to the dry binder employed (Fig. 1). An increase in the concentration of glyceryl dibehenate to $15 \%$ in the tableting material with microcrystalline cellulose causes a decrease in the strength of tablets; on the other hand, its increase to $20 \%$ increases the 
Table II. Energy profile values of compression and plasticity

\begin{tabular}{ccccccc}
\hline $\begin{array}{c}\text { Tableting } \\
\text { material }\end{array}$ & $\begin{array}{c}\mathrm{CF} \\
(\mathrm{kN})\end{array}$ & $E_{\max } \pm \mathrm{SD}(\mathrm{J})$ & $E_{1} \pm \mathrm{SD}(\mathrm{J})$ & $E_{2} \pm \mathrm{SD}(\mathrm{J})$ & $E_{3} \pm \mathrm{SD}(\mathrm{J})$ & $\mathrm{PI} \pm \mathrm{SD}(\%)$ \\
\hline MCC + & 4 & $8.84 \pm 0.14$ & $5.31 \pm 0.15$ & $3.10 \pm 0.02$ & $0.43 \pm 0.01$ & $87.9 \pm 0.2$ \\
$10 \% \mathrm{C}$ & 5 & $11.72 \pm 0.53$ & $7.38 \pm 0.52$ & $3.73 \pm 0.04$ & $0.61 \pm 0.01$ & $86.0 \pm 0.2$ \\
& 6 & $15.27 \pm 0.32$ & $10.18 \pm 0.30$ & $4.28 \pm 0.04$ & $0.81 \pm 0.01$ & $84.1 \pm 0.2$ \\
\hline MCC + & 4 & $8.35 \pm 0.14$ & $5.23 \pm 0.14$ & $2.71 \pm 0.02$ & $0.42 \pm 0.01$ & $86.6 \pm 0.2$ \\
$15 \% \mathrm{C}$ & 5 & $10.75 \pm 0.30$ & $6.92 \pm 0.29$ & $3.24 \pm 0.03$ & $0.60 \pm 0.01$ & $84.5 \pm 0.2$ \\
& 6 & $13.42 \pm 0.37$ & $8.93 \pm 0.35$ & $3.68 \pm 0.04$ & $0.81 \pm 0.01$ & $82.0 \pm 0.2$ \\
\hline & 4 & $8.47 \pm 0.14$ & $5.34 \pm 0.15$ & $2.71 \pm 0.02$ & $0.42 \pm 0.00$ & $86.6 \pm 0.1$ \\
$\mathrm{MCC}+$ & 5 & $10.96 \pm 0.20$ & $7.17 \pm 0.18$ & $3.19 \pm 0.03$ & $0.60 \pm 0.01$ & $84.2 \pm 0.2$ \\
$20 \% \mathrm{C}$ & 6 & $13.13 \pm 0.39$ & $8.73 \pm 0.35$ & $3.56 \pm 0.06$ & $0.80 \pm 0.01$ & $81.7 \pm 0.3$ \\
\hline & 12 & $18.21 \pm 0.22$ & $11.74 \pm 0.23$ & $3.78 \pm 0.02$ & $2.70 \pm 0.01$ & $58.3 \pm 0.2$ \\
FL + & 14 & $22.07 \pm 0.37$ & $14.22 \pm 0.34$ & $4.24 \pm 0.07$ & $3.61 \pm 0.02$ & $54.0 \pm 0.3$ \\
$10 \% \mathrm{C}$ & 16 & $26.05 \pm 0.23$ & $16.80 \pm 0.26$ & $4.68 \pm 0.05$ & $4.57 \pm 0.02$ & $50.6 \pm 0.4$ \\
\hline & 12 & $18.59 \pm 0.17$ & $12.43 \pm 0.18$ & $3.40 \pm 0.02$ & $2.76 \pm 0.02$ & $55.2 \pm 0.3$ \\
FL + & 14 & $22.25 \pm 0.35$ & $14.80 \pm 0.33$ & $3.81 \pm 0.08$ & $3.65 \pm 0.04$ & $51.1 \pm 0.3$ \\
$15 \% \mathrm{C}$ & 16 & $26.14 \pm 0.45$ & $17.38 \pm 0.42$ & $4.13 \pm 0.02$ & $4.64 \pm 0.03$ & $47.1 \pm 0.1$ \\
\hline & 12 & $18.64 \pm 0.34$ & $12.63 \pm 0.33$ & $3.21 \pm 0.05$ & $2.80 \pm 0.02$ & $53.5 \pm 0.3$ \\
FL + & 14 & $21.84 \pm 0.18$ & $14.64 \pm 0.17$ & $3.53 \pm 0.03$ & $3.67 \pm 0.03$ & $49.0 \pm 0.2$ \\
$20 \% \mathrm{C}$ & 16 & $25.60 \pm 0.49$ & $17.09 \pm 0.48$ & $3.83 \pm 0.02$ & $4.68 \pm 0.02$ & $45.0 \pm 0.1$ \\
\hline
\end{tabular}

MCC - Microcel MC-102

C-Compritol 888 ATO

FL - Flowlac 100

$\mathrm{CF}$ - compression force

$\mathrm{E}_{\max }$ - total energy

SD - standard deviation

$\mathrm{E}_{1}$-energy of precompression

$E_{2}$-energy of plastic deformation

$\mathrm{E}_{3}$ - energy of elastic deformation

$\mathrm{Pl}$ - plasticity

strength. This fact is connected with the initial decrease in the bonds of microcrystalline cellulose, a further increase in glyceryl dibehenate concentration compensating decreased binding ability (12). In the case of spray-dried lactose, it depends on compression force, because in the case of compression forces of 12 and $14 \mathrm{kN}$ increasing concentration of glyceryl dibehenate does not influence the strength of tablets. Only at $16 \mathrm{kN}$ there is a decrease in the strength of tablets due to addition of $20 \%$ glyceryl dibehenate (13).

\section{Evaluation of tablet dissolution}

Active substance release from tablets was described by kinetic models, in which the amount of drug dissolved $\left(A_{\mathrm{t}}\right)$ is a function of the dissolution time $t, A_{\mathrm{t}}=\mathrm{f}(t)$. To analyze 


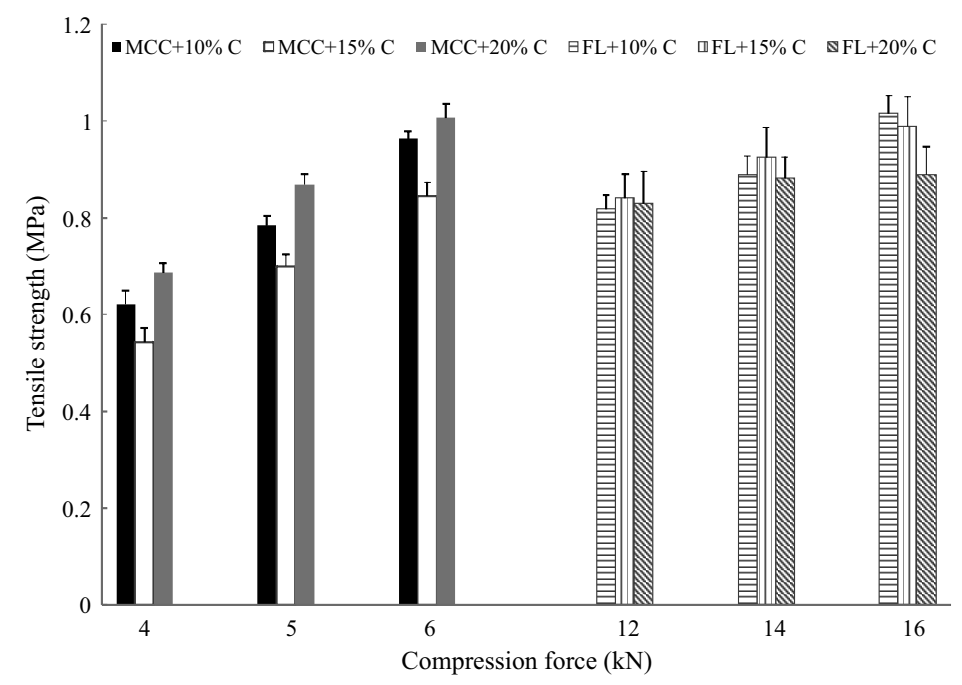

Fig. 1. Tensile strength of tablets as a function of compression force (mean $\pm \mathrm{SD}, n=10)(\mathrm{MCC}-\mathrm{Mi}$ crocel MC-102, C - Compritol 888 ATO, FL - Flowlac 100).

the dissolution profiles of tablets with the different dry binders, the obtained experimental curves were fitted by the first-order, Weibull (Fig. 2) and Higuchi models (14). Detailed regression and statistical analysis of the effect of the employed dry binder on drug release was carried out. The coefficient of determination $\left(R^{2}\right)$, residual sum of squares $(R S S)$, amount of active substance which will be released in infinite tim $\left(A_{\infty}\right)$, release rate constant

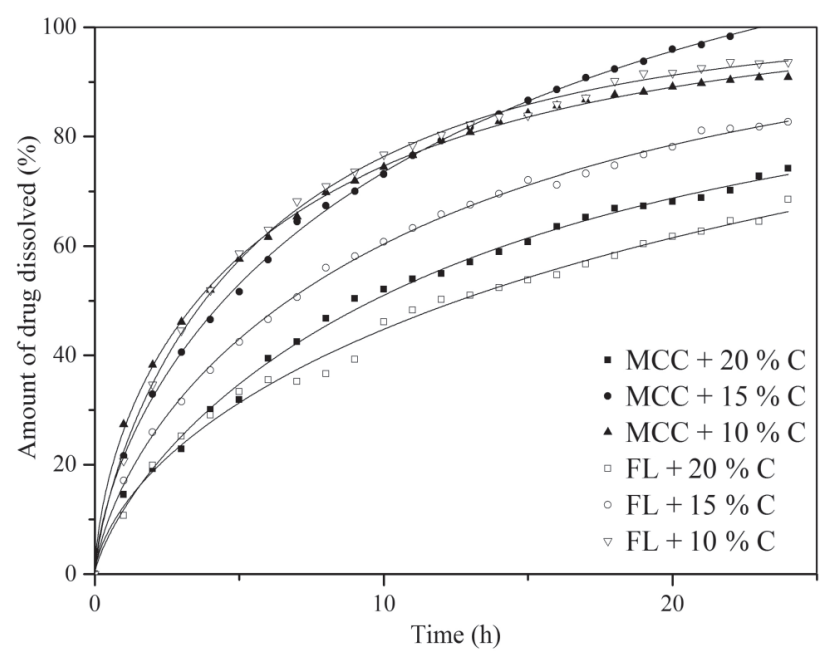

Fig. 2. Release of the active substance from investigated formulations fitted by the Weibull model (mean \pm SD, $n=6$ ) (MCC - Microcel MC-102, C - Compritol 888 ATO, FL - Flowlac 100). 
with standard deviation $(k)$ and Higuchi dissolution constant $\left(k_{\mathrm{H}}\right)$ were estimated based on non-linear regression analysis using the least-squares method (LSM). Results of the regression analysis of dissolution profiles (Table III) confirm the highest tightness of fit (the highest value of $R^{2}$ and the lowest value of $R S S$ ) for the Weibull model. The shape parameter in the Weibull model $(n)$ is lower than 1 for all the prepared dosage forms, which indicates a higher slope of the initial part of the dissolution curve in comparison with the $1^{\text {st }}$-order exponential profile. Order of the release process (corresponds to the shape parameter in the Weibull model) of the active substance from the investigated forms is in the range from 0.64 to 0.77 , which proves that more factors affect the release rate. The release rate of the active substance decreases with increasing content of glyceryl dibehenate. Nonlinear regression analysis of the rate constant mean values (Table III) for the drug formulation with the same content of glyceryl dibehenate showed that there were insignificant statistical differences between tablets with microcrystalline cellulose and spray-dried lactose. Analysis of the amount of drug dissolved (mean values) showed insignificant differences between tablets with microcrystalline cellulose and spray-dried lactose containing $10 \%$ of glyceryl dibehenate but more significant differences for formulations with 15 and $20 \%$ of glyceryl dibehenate. As shown in Fig. 2, increasing content of glyceryl dibehenate in tablets with spray-dried lactose decreases the $A_{\infty}$ value. Formulations containing microcrystalline cellulose and $20 \%$ of glyceryl dibehenate (MCC $+20 \% \mathrm{C}$ ) show lower $A_{\infty}$ value in comparison with tablets containing $10 \%$ of glycerol dibehenate (MCC $+10 \% \mathrm{C})$. For tablets containing microcrystalline cellulose and $15 \%$ of glyceryl dibehenate (MCC $+15 \%$ C), an anomalous dissolution profile (should be the subject of further research) was found.

Table III. Dissolution data by non-linear regression analysis

\begin{tabular}{|c|c|c|c|c|c|c|c|c|c|}
\hline \multirow[t]{2}{*}{$\begin{array}{l}\text { Tableting } \\
\text { material }\end{array}$} & \multicolumn{3}{|c|}{$\begin{array}{c}1^{\text {th }} \text { order kinetic model } \\
\qquad A_{\mathrm{t}}=A_{\propto}\left(1-e^{-k t}\right)\end{array}$} & \multicolumn{4}{|c|}{$\begin{array}{c}\text { Weibull model } \\
A_{\mathrm{t}}=A_{\propto}\left(1-e^{-(k t)^{n}}\right)\end{array}$} & \multicolumn{2}{|c|}{$\begin{array}{l}\text { Higuchi model } \\
\qquad A_{\mathrm{t}}=k_{\mathrm{H}} \sqrt{t}\end{array}$} \\
\hline & $k \times 10^{3}\left(\mathrm{~h}^{-1}\right)^{\mathrm{a}}$ & $R^{2}$ & $R S S$ & $k \times 10^{3}\left(\mathrm{~h}^{-n}\right)^{\mathrm{a}}$ & $R^{2}$ & $R S S$ & $n$ & $R^{2}$ & $R S S$ \\
\hline $\begin{array}{c}\text { FL + } \\
10 \% \mathrm{C}\end{array}$ & $197.0 \pm 7.7$ & 0.9890 & 153.00 & $162.1 \pm 6.1$ & 0.9984 & 20.02 & 0.75 & 0.9372 & 549.43 \\
\hline $\begin{array}{c}\mathrm{FL}+ \\
15 \% \mathrm{C}\end{array}$ & $140.5 \pm 6.7$ & 0.9875 & 141.69 & $85.6 \pm 7.7$ & 0.9985 & 15.55 & 0.71 & 0.9860 & 158.47 \\
\hline $\begin{array}{c}\mathrm{FL}+ \\
20 \% \mathrm{C}\end{array}$ & $114.2 \pm 9.0$ & 0.9723 & 203.90 & $71.6 \pm 7.1$ & 0.9918 & 54.82 & 0.64 & 0.9921 & 58.41 \\
\hline $\begin{array}{l}\mathrm{MCC}+ \\
10 \% \mathrm{C}\end{array}$ & $212.1 \pm 1.2$ & 0.9760 & 292.21 & $151.5 \pm 5.9$ & 0.9993 & 7.85 & 0.64 & 0.9456 & 661.96 \\
\hline $\begin{array}{l}\mathrm{MCC}+ \\
15 \% \mathrm{C}\end{array}$ & $143.6 \pm 7.7$ & 0.9835 & 270.88 & $72.2 \pm 5.6$ & 0.9994 & 8.84 & 0.66 & 0.9881 & 195.96 \\
\hline $\begin{array}{l}\mathrm{MCC}+ \\
20 \% \mathrm{C}\end{array}$ & $117.4 \pm 5.5$ & 0.9903 & 91.41 & $76.5 \pm 12.0$ & 0.9958 & 36.56 & 0.77 & 0.9889 & 104.19 \\
\hline
\end{tabular}

MCC - Microcel MC-102

C - Compritol 888 ATO

FL - Flowlac 100

${ }^{\text {a }}$ Mean \pm SD, $n=6$. 


\section{CONCLUSIONS}

Glyceryl dibehenate influences compressibility of tableting materials with microcrystalline cellulose more than those with spray-dried lactose. Glyceryl dibehenate in tableting materials with microcrystalline cellulose decreases the total energy of compression while in a higher concentration it increases the strength of tablets. The release rate constant of salicylic acid from tablets is decreased with increasing concentrations of glyceryl dibehenate, but it is not significantly different within the range of contents of the dry binders used. Increasing the content of glyceryl dibehenate influences the $A_{\infty}$ value in all formulations.

Acknowledgments. - The study was supported by the firms Meggle Pharma, Blanver and SPI Pharma, which supplied the samples of the excipients tested.

\section{REFERENCE}

1. I. Singh, P. Kumar, N. Rani and V. Rana, Investigation of different lipid based materials as matrices designed to control the release of a hydrophobic drug, Int. J. Pharm. Sci. Drug Res. 1 (2009) 158-163.

2. GATTEFOSSÉ, Lipid excipients for oral dosage forms, Documents for Oral route. http://www.gattefosse. com/en/document-center/; last access July 15, 2013.

3. S. H. A. El-Halim, M. M. Amin, O. N. El-Gazayerly and N. A. A. El-Gavad, Comparative study on the different techniques for the preparation of sustained-release hydrophobic matrices of a highly water-soluble drug, Drug Discov. Ther. 4 (2010) 484-492.

4. L. M. E. McIndoe, Glyceryl Dibehenate, in Handbook of Pharmaceutical Excipients (Eds. R. C. Rowe, P. J. Sheskey and S. C. Owen), $5^{\text {th }}$ ed., Pharmaceutical Press, Washington 2006, pp. 304-305.

5. GATTEFOSSÉ, Developing sustained release tablets with Compritol ${ }^{\circledR} 888$ ATO, Formulation Guidelines, Version 2, Lyon, 2010.

6. G. Ragnarsson, Force-displacement and Network Measurements, in Pharmaceutical Powder Compaction Technology (Eds. G. Alderborn and Ch. Nyström, Marcel Dekker Inc., New York 1996, pp. 77-96.

7. A. Stamm and C. Mathis, Verpressbarkeit von Festen Hilfsstoffen für Direkttablettierung, Acta Pharm. Technol. 22 (1976) 7-16.

8. J. T. Fell and J. M. Newton, Determination of tablet strength by diametral-compression test, J. Pharm. Sci. 59 (1970) 688-691; DOI: 10.1002/jps.2600590523.

9. European Pharmacopoeia, $7^{\text {th }}$ ed., Vol. 1, Council of Europe, Strasbourg 2010, pp. 256-263.

10. V. A. Belousov, Choice of optimal pressure values in tableting medicinal powders, Khim. Farm. Zh. 10 (1976) 105-111.

11. B. A. C. Carlin, Direct Compression and the Role of Filler Binders, in Pharmaceutical Dosage Forms: Tablets, Eds. L. L. Augsburger and S. W. Hoag), $3^{\text {th }}$ ed., Informa Healthcare USA, Inc., New York 2008, Vol. 2, pp. 173-216.

12. N. O. Iloanusi and J. B. Schwartz, The effect of wax on compaction of microcrystalline cellulose beads made by extrusion and spheronization, Drug Dev. Ind. Pharm. 24 (1998) 37-44; DOI: 10.3109/03639049809082350.

13. M. Roberts, D. Vellucci, S. Mostafa, C. Miolane and D. Marchaud, Development and evaluation of sustained-release Compritol ${ }^{\circledR} 888$ ATO matrix mini-tablets, Drug Dev. Ind. Pharm. 38 (2012) 10681076; DOI: 10.3109/03639045.2011.638302.

14. P. Costa and J. M. Sousa Lobo, Modeling and comparison of dissolution profiles, Eur. J. Pharm. Sci. 13 (2001) 123-133; DOI: 10.1016/S0928-0987(01)00095-1. 